\title{
Effects of Form Birefringence on Flow Birefringence of Polymer Solutions in Semidilute Regions
}

\author{
Yoshiaki TAKAHASHI, Masayoshi YAMAGUCHI, Koichiro HORI,* \\ Fumitoshi SUZUKI, ${ }^{* *}$ and Ichiro NODA \\ Department of Synthetic Chemistry, Nagoya University, Furo-cho, \\ Chikusa-ku, Nagoya 464-01, Japan \\ * Mitsubishi Electric Corporation, 4-1, Mizuhara, Itami, \\ Hyogo 664, Japan \\ ** Nippon Zoen Co., Ltd., 1-2-1, Yako, Kawasaki-ku, \\ Kawasaki, Kanagawa 210, Japan
}

(Received July 6, 1989)

\begin{abstract}
Effects of the form birefringence on flow birefringence of polymer solutions in semidilute region were studied by using polystyrenes in bromobenzene and toluene, which are matching and non-matching solvents, respectively. It was found that the contribution of the form birefringence rapidly decreases when the degree of coil-overlapping becomes higher than the crossover point from dilute to semidilute regions for thermodynamic properties.

KEY WORDS Flow Birefringence / Form Birefringence / Intrinsic Birefringence / Semidilute Solution / Dilute Solution / Polystyrene /
\end{abstract}

The orientation and deformation of polymer chains in solutions and melts under a flow lead to an optical anisotropy of the polymer systems called flow birefringence. ${ }^{1,2}$ From the flow birefringence measurements we can obtain the birefringence $\Delta n$, which is the refractive index difference between the two principal axis, and the extinction angle $\chi$, which is the angle between the principal axis and the flow direction.

It is well known that the flow birefringence of polymer solutions consists of two parts, i.e., the intrinsic birefringence $\Delta n^{\mathrm{i}}$ and the form birefringence $\Delta n^{\mathrm{f} 1-3}$

$$
\Delta n=\Delta n^{\mathrm{i}}+\Delta n^{\mathrm{f}}
$$

The intrinsic birefringence is the contribution from the anisotropic polarizability of polymer segments oriented under the flow, while the form birefringence is the contribution from the anisotropical internal field caused by the anisotropy of the shape of polymer coil when the refractive index of polymer chain is different from that of solvent.

Since there is no form birefringence in uniform systems such as in concentrated solutions and in melts, only the intrinsic birefringence is observed so that the observed birefringence and extinction angle are directly related to the shear stress $P_{12}$ and the primary normal force difference $P_{11}-P_{22}$, that is, we have the stress-optical law ${ }^{1,2}$

$$
\begin{aligned}
& P_{12}-\eta_{\mathrm{s}} \dot{\gamma}=\Delta n \cdot \sin 2 \chi / 2 C \\
& P_{11}-P_{22}=\Delta n \cdot \cos 2 \chi / C
\end{aligned}
$$

where $\eta_{\mathrm{s}}$ is the solvent viscosity, $\dot{\gamma}$ is the shear rate and $C$ is the stress-optical coefficient.

When the shear rate is low, the birefringence $n_{12}=\Delta n \cdot \sin 2 \chi$ is proportional to shear rate and the proportionality factor $\lambda_{\mathrm{M}}=n_{12} / \dot{\gamma}$ is called the Maxwell constant. ${ }^{1-3}$

At an infinite dilution, we define the intrinsic Maxwell constant as 


$$
[n]=\lim _{c \rightarrow 0} \lambda_{\mathrm{M}} / c \eta_{\mathrm{s}}
$$

According to the theories, ${ }^{1-3}$ the intrinsic Maxwell constant of polymer solution is given by

$$
\begin{aligned}
{[n] } & =\left[n^{\mathrm{i}}\right]+\left[n^{\mathrm{f}}\right] \\
& =2 C[\eta]+k_{\mathrm{f}}(\partial n / \partial c)^{2}(M / n R T)
\end{aligned}
$$

where the superscripts $\mathrm{i}$ and $\mathrm{f}$ denote the intrinsic and form birefringences, respectively, $n$ is the refractive index of solvent, $\partial n / \partial c$ is the refractive index increment and $k_{\mathrm{f}}$ is a numerical constant $(\sim 0.34){ }^{2)}$ It was reported ${ }^{2)}$ that eq 3 well explains experimental data at an infinite dilution. As seen in eq 4 the effects of the form birefringence can be eliminated and the stress-optical law is valid even in dilute solutions if we use a matching solvent, in which the refractive index increment $\partial n / \partial c$ is negligibly small.

Even if the refractive index increment is not small, the contribution of form birefringence is negligibly small in concentrated solutions, as menioned before, while the contribution is large in dilute solutions. However, it is not clear how much the form birefringence contributes to the flow birefringence in semidilute solutions, where polymer chains extensively overlap each other, but the segment concentration is still small.

The flow birefringence method is one of the most powerful technique for measuring viscoelastic properties of polymers in dilute and semidilute solutions if the stress-optical law is valid, since the sensitivity of this method is much higher than that of mechanical methods for measuring viscoelastic properties owing to the optical detection.

Therefore, it is important to study the applicability of the stress-optical law in semidilute solutions when the refractive index increment is not small. In this work, thus, we studied the contribution of the form birefringence of polystyrene in toluene, which is a non-matching solvent, in terms of degree of coil-overlapping. The contribution of the form birefringence is discussed in terms of $n_{12}=\Delta n \cdot \sin 2 \chi$ which corresponds to shear stress as shown in eq $2 \mathrm{a}$. The observed $\chi$ values are not simply related to $\chi$ in eq $2 \mathrm{a}$ when the form birefringence exists. $^{1,2}$ In this work, however, we use the observed $\chi$ values, since our primary aim is to know the critical concentrations where the effects of form birefringence disappear. Therefore, our discussion is limited in the linear region of deformation so that the observed $\chi$ values are expected to be close to $\chi$ in eq $2 \mathrm{a}$ especially in the neighbourhood of the critical concentrations. The intrinsic birefringence data were directly measured in a matching solvent, bromobenzene or they were calculated from viscosity data using eq $2 a$.

\section{EXPERIMENTAL}

Samples used here were two linear polystyrenes with narrow molecular weight distributions, F-80 and F-450, purchased from Tosoh Corporation. Their weight average molecular weights are $7.75 \times 10^{5}$ and $4.48 \times 10^{6}$, respectively.

The critical concentration $c^{*}$ for polymer coil-overlapping is defined by

$$
c^{*} \equiv 3 M /\left(4 \pi\left\langle s^{2}\right\rangle^{3 / 2} N_{\mathrm{A}}\right)
$$

where $M$ and $\left\langle s^{2}\right\rangle$ are the molecular weight and the mean square radius of gyration of polymer, respectively, and $N_{\mathrm{A}}$ is Avogadro's number. The $c^{*}$ for F-80 and F-450 in good solvents are 0.580 and $0.154 \mathrm{~g} \mathrm{dl}^{-1}$, respectively. ${ }^{4}$

Solvents used here were bromobenzene and toluene, which are good solvents for polystyrene. The former is practically a matching solvent for polystyrene $(\partial n / \partial c=0.046$ $\left.\mathrm{cm}^{3} \mathrm{~g}^{-1}\right)$, while the latter is not $(\partial n / \partial c=0.106$ $\left.\mathrm{cm}^{3} \mathrm{~g}^{-1}\right){ }^{5}$

The flow birefringence measurements were carried out for polystyrene samples F-80 and $\mathrm{F}-450$ in toluene, and for F-450 in bromoben- 
zene at room temperatures $\left(20-25^{\circ} \mathrm{C}\right)$ with an apparatus designed and constructed in this laboratory. $^{6}$ In the optical system on this apparatus the circularly polarized laser beam with $633 \mathrm{~nm}$ of wavelength is employed as the incident light. The birefringence $\Delta n$ and the extinction angle $\chi$ were evaluated from the displacement and the rotation angle of micrometer-driven Babinet-Soleil compensation plate. The details of the apparatus and the measurement methods were described in previous papers. ${ }^{6,7}$ Birefringence data of F-450 in bromobenzene were already reported in a previous paper. ${ }^{7}$

The zero-shear viscosity $\eta^{\circ}$ of sample F-80 in toluene at $30^{\circ} \mathrm{C}$ was measured by a capillary viscometer of Maron-Krieger-Sisko type as reported in a previous paper. ${ }^{8}$

\section{RESULTS}

Figure 1 shows double logarithmic plots of the total birefringence data $n_{12} / \eta_{\mathrm{s}}$ of sample F80 in toluene against shear rate $\dot{\gamma}$. The data at each concentration can be represented by straight lines with the slopes of unity within the experimental error, indicating that all the data are obtained in the linear region of deformation. Solid lines denote the intrinsic birefringence $n_{12} \mathrm{i} / \eta_{\mathrm{s}}$ calculated from the zeroshear viscosity data assuming the stress-optical coefficient for polystyrene in bromobenzene, $C=5.75 \times 10^{-10} \mathrm{~cm}^{2} \mathrm{dyn}^{-1}{ }^{3}$ It can be seen in this figure that the birefringence data in toluene and the calculated values from viscosity coincide with each other at the highest concentration, but the data in toluene deviate from the calculated intrinsic values with decreasing the concentration.

Figure 2 shows the same kind of plot for sample F-450 as in Figure 1. Open squares denote the total birefringence data $n_{12} / \eta_{\mathrm{s}}$ in toluene while filled squares denote intrinsic birefringence data $n_{12}{ }^{\mathrm{i}} / \eta_{\mathrm{s}}$ measured in bromobenzene except for the data at the highest concentration ( $\mathbf{L})$, which are calculated from

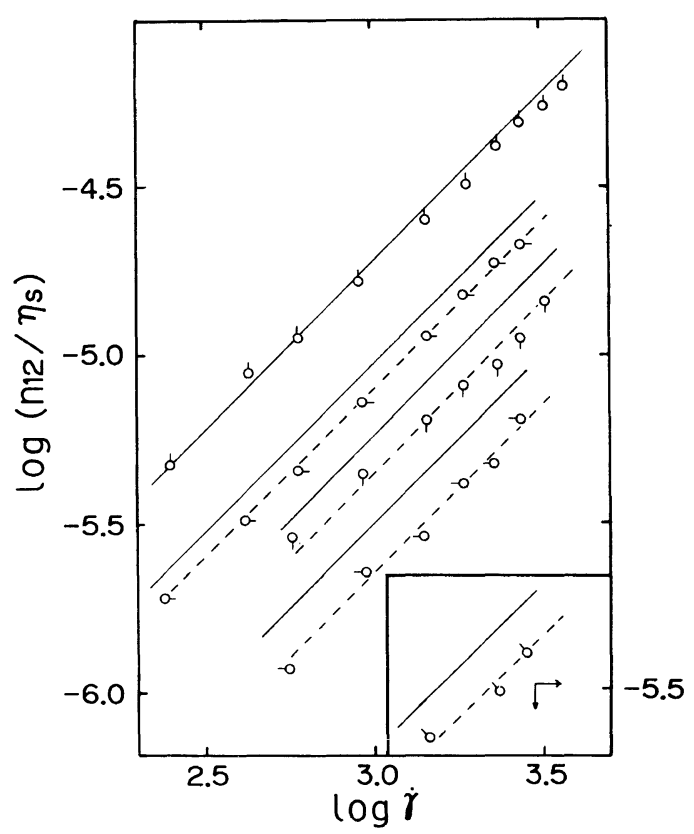

Figure 1. Double logarithmic plots of total birefringence $n_{12} / \eta_{\mathrm{s}} v s$. shear rate $\dot{\gamma}$ for sample $\mathrm{F}-80$ in toluene. Symbols $\bigcirc, O^{-}, \bigcirc,-\bigcirc$, and $\bigcirc$ denote the data for $2.66,1.85,1.36,0.924$, and $0.692 \mathrm{~g} \mathrm{dl}^{-1}$, respectively. Solid lines denote the corresponding intrinsic birefringence data calculated from the viscosity data. ${ }^{4}$

the viscosity data reported in a previous work. ${ }^{8}$ Although the data scatter somewhat more than the data for F-80 in Figure 1, it is apparent that the $n_{12} / \eta_{\mathrm{s}}$ data in toluene coincide with the $n_{12}{ }^{\mathrm{i}} / \eta_{\mathrm{s}}$ data at high concentrations but deviate from them with decreasing the concentration.

The form birefringence $n_{12}{ }^{\mathrm{f}}=\Delta n^{\mathrm{f}} \cdot \sin 2 \chi$ in toluene can be evaluated from the difference between the total birefringence $n_{12}$ and the intrinsic birefringence $n_{12}{ }^{\mathrm{i}}$. Actually, the refractive index increment of polystyrenebromobenzene solution is very small but not zero as described before. Considering that the form birefringence is proportional to $(\partial n / \partial c)^{2}$, therefore, the actual form birefringences in the absolute value must be about $20 \%$ larger than those evaluated from the bromobenzene solutions. Moreover, it should be noted that the birefringence data are not linear to $\dot{\gamma}$ at high $\dot{\gamma}$ 


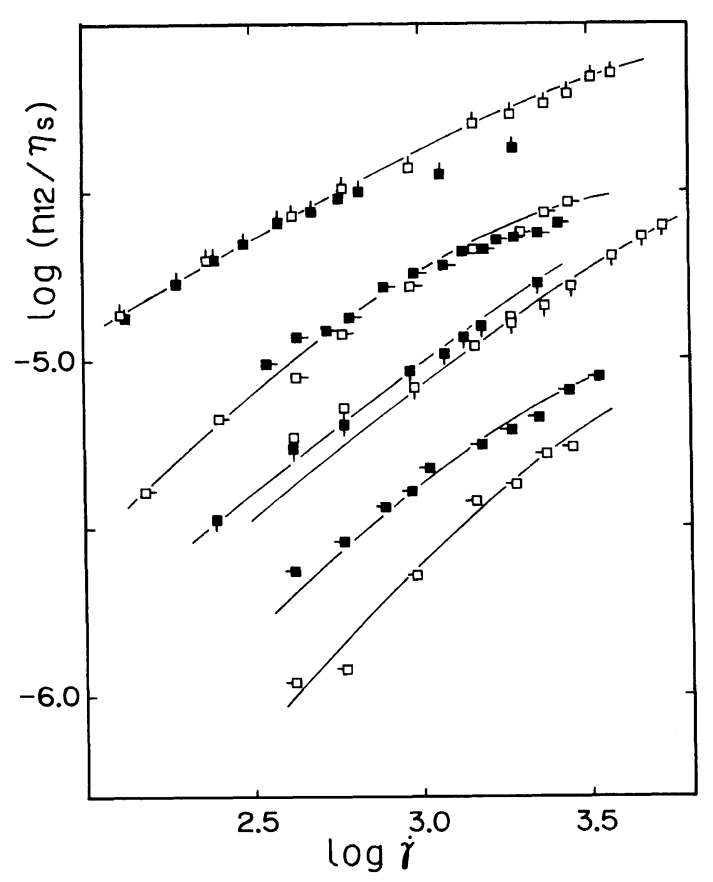

Figure 2. Double logarithmic plots of total birefringence $n_{12} / \eta_{\mathrm{s}}$ vs. shear rate $\dot{\gamma}$ for sample F-450 in toluene. Symbols $\square, \square, \square$, and $-\square$ denote the data for $1.58,0.96,0.64$, and $0.34 \mathrm{~g} \mathrm{dl}^{-1}$, respectively. The corresponding filled symbols denote the data of intrinsic birefringence measured in bromobenzene.

in Figure 2. The dependences of intrinsic and form birefringences on shear rate may be different. Further works are needed to clarify the shear rate dependences.

\section{DISCUSSION}

The total Maxwell constant $\lambda_{\mathrm{M}}$, and the contributions from intrinsic and form birefringences, $\lambda_{\mathrm{M}}{ }^{\mathrm{i}}$ and $\lambda_{\mathrm{M}}{ }^{\mathrm{f}}$, can be evaluated from the data in the linear regions in Figures 1 and 2. Figure 3 shows double logarithmic plots of $\lambda_{\mathrm{M}}{ }^{\mathrm{f}} / \eta_{\mathrm{s}}$ and $\lambda_{\mathrm{M}}{ }^{\mathrm{i}} / \eta_{\mathrm{s}}$ against $c$. It can be seen that the intrinsic terms increase steeply as viscosity, while the form terms increase slightly and tend to level off at the higher concentrations than $2 c^{*}$. Hence, the difference between the intrinsic and form birefringences becomes larger than one order at the higher concentrations. From

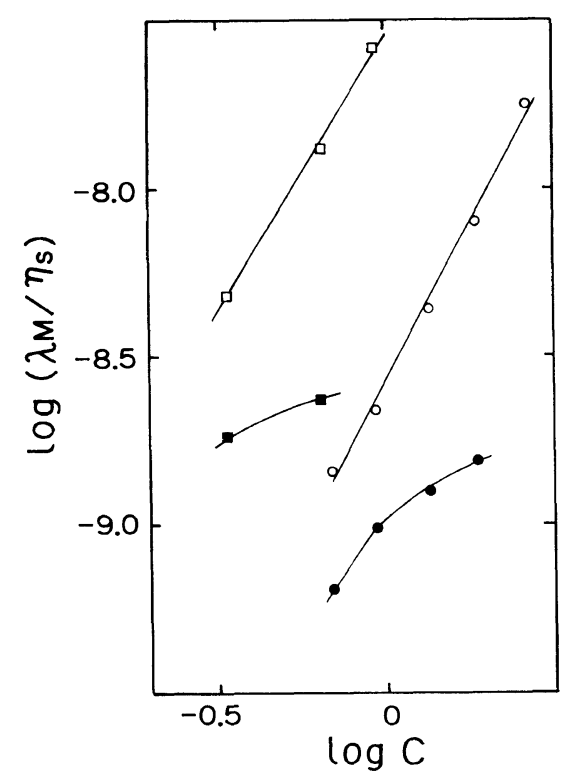

Figure 3. Double logarithmic plots of $\left(\lambda_{\mathrm{M}} / \eta_{\mathrm{s}}\right)$ vs. $c$. Open squares and circles denote the intrinsic data of samples F-450 and F-80, respectively. Filled symbols denote the corresponding form data.

the data at low concentrations, the limiting value of $\left[n^{\mathrm{f}}\right] / M=\lim _{c \rightarrow 0} \lambda_{\mathrm{M}} \mathrm{f}^{\mathrm{f}} / \eta_{\mathrm{s}} c M$ can be estimated. The estimated value is $1.2 \times 10^{-13}$ which is about $25 \%$ different from the calculated value by eq 4 with $k_{\mathrm{f}}=0.34$. This difference may partly due to scarcity of the data and experimental error at low concentrations. However, almost the same relative deviation was reported for other experiments. ${ }^{2}$

According to Doi and Edwards, ${ }^{3}$ the Maxwell constant for form birefringence of polymer chains in semidilute solutions is given by

$$
\lambda_{\mathrm{M}}{ }^{\mathrm{f}} \simeq N_{\mathrm{A}} \eta_{\mathrm{s}} c \xi^{3} c(\partial n / \partial c)^{2} / n R T
$$

where $\xi$ is the correlation length. Dividing the above equation with $\left[n^{\mathrm{f}}\right]$ in eq 4 , we have the reduced form birefringence as

$$
\left(\lambda_{\mathrm{M}}{ }^{\mathrm{f}} / c \eta_{\mathrm{s}}\right) /\left[n^{\mathrm{f}}\right] \propto c \xi^{3} N_{\mathrm{A}} / \boldsymbol{M}
$$

According to the scaling theory of semidilute solutions, ${ }^{10,11}$ we have 


$$
c \xi^{3} N_{\mathrm{A}} / M \simeq M_{\xi} / M=\left(\xi^{2} /\left\langle s^{2}\right\rangle\right)^{1 / 2 v}
$$

where $M_{\xi}$ is the molecular weight of a chain whose length is equal to $\xi$ and $v$ is the excluded volume exponent in the relationship between mean square radius of gyration and molecular weight, $\left\langle s^{2}\right\rangle \propto M^{2 v}$. Moreover, $\xi^{2} /\left\langle s^{2}\right\rangle$ is given by the universal function of $c / c^{*} \mathrm{as}^{9,10}$

$$
\xi^{2} /\left\langle s^{2}\right\rangle \propto\left(c / c^{*}\right)^{2 v /(1-3 v)}
$$

Introducing eq 8 and 9 into eq 7 we have

$$
\lambda_{\mathrm{M}}^{\mathrm{f}} / c \eta_{\mathrm{s}}\left[n^{\mathrm{f}}\right] \propto\left(c / c^{*}\right)^{1 /(1-3 v)}
$$

Double logarithmic plots of the reduced form birefringence against degree of coil overlapping $c / c^{*}$ are shown in Figure 4. In this figure, the data in dilute region $\left(c / c^{*}<1\right)$ reported by Janeschitz-Kriegl ${ }^{2}$ are also shown. The experimental values of $\left[n^{\mathrm{f}}\right]$ are used to analyze all these data because there are discrepancies between experimental and calculated values as mentioned above. The reduced data are almost constant up to 3 for $c / c^{*}$ as shown by straight line. Tsvetkov ${ }^{1}$ used a different kind of reduced plot for form birefringence of various polymer-solvent systems, in which $\lambda_{\mathrm{M}}{ }^{\mathrm{f}} / \eta_{\mathrm{s}} c\left[n^{\mathrm{f}}\right]$ is devided by the reduced zero-shear viscosity $\left(\eta^{\circ}-\eta_{\mathrm{s}}\right) / \eta_{\mathrm{s}} c[\eta]$, and reported that the reduced plots become a universal function of $c[\eta]$ up to $c[\eta]=2$. Since the reduced zero-shear viscosity is also a universal function of $c / c^{*}$, which is almost equal to $c[\eta]{ }^{8}$ this reduced form can be easily converted to our reduced form. These data thus obtained are consistent with our data in Figure 4.

It is not easy to evaluate the form birefringence precisely in semidilute solutions, because the intrinsic birefringence sharply increases in this region as shown in Figure 3. In our experiments only one experimental point obviously deviates from the straight line as shown in Figure 4. Therefore, it is difficult to compare the data with the theory objectively. However, the experimental data can be fitted with a line having the slope $(-1.25)$ calculated from eq 10 using $v=0.6$ as shown by the

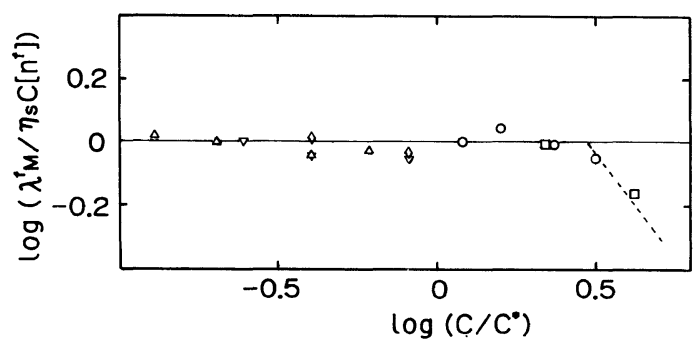

Figure 4. Double logarithmic plots of $\lambda_{\mathrm{M}}{ }^{\mathrm{f}} / \eta_{\mathrm{s}} c\left[n^{\mathrm{f}}\right] v s$. $c / c^{*}$. Symbols $\triangle, \nabla$, and $\diamond$ denote the data in cyclohexanone, dioxane, and chlorobenzene, respectively. ${ }^{2}$ Other symbols are the same as in Figure 3. Dotted line denote the slope calculated from eq 10 .

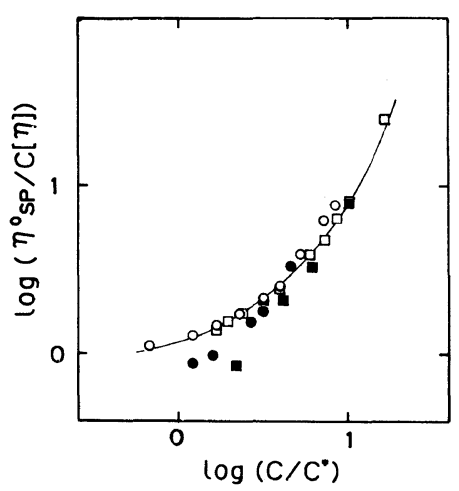

Figure 5. Double logarithmic plots of $\eta_{\mathrm{sp}}^{\circ} / c[\eta] v s . c / c^{*}$. Squares and circles denote the data for samples F-450 and F-80, respectively. ${ }^{4}$ Filled symbols denote the corresponding data obtained from flow birefringence in toluene.

broken line in Figure 4 , by assuming that the deviation occurs around $c / c^{*}=3$. It is interesting that $3 c^{*}$ is slightly higher than the crossover point from dilute to semidilute solution regions for thermodynamic properties. ${ }^{11,12}$

In Figure 5 apparent zero-shear viscosities evaluated from the flow birefringence data in toluene are compared with the observed zeroshear viscosities ${ }^{4}$ in the reduced form of $\eta_{\mathrm{sp}}^{\circ} /$ $c[\eta] v s . c / c^{*} \cdot 4,8$ It is clear that the viscosity data evaluated from flow birefringence in toluene deviate from the observed viscosity data at the low $c / c^{*}$ values owing to the form birefringence, but the both coincide with each other at the higher $c / c^{*}$ values than 4 .

In summary we can conclude that the contri- 
bution of the form birefringence to the flow birefringence rapidly decreases as the degree of coil-overlapping becomes slightly higher than the cross-over point from dilute to semidilute solutions for thermodynamic properties, and hence the stress-optical law becomes completely valid in semidilute region for viscosity, even if the refractive index increment is large, since the cross-over concentration from dilute to semidilute solutions for viscosity is about 5 times higher than that of thermodynamic properties in good solvents. ${ }^{4}$

\section{REFERENCES}

1. V. N. Tsvetkov, "Newer methods of Polymer Characterization," B. Ke, Ed., Interscience, New York, N. Y., 1964, p 563.

2. H. Janeschitz-Kriegel, Adv. Polym. Sci., 6, 170 (1969).

3. M. Doi and S. F. Edwards, "The Theory of Polymer
Dynamics," Oxford University Press, Oxford, 1986, Chapters 4 and 5.

4. Y. Takahashi, I. Noda, and M. Nagasawa, Macromolecules, 18, 2220 (1985).

5. J. Brandrup and E. H. Immergut, Ed., "Polymer Handbook," 2nd ed, Wiley-Interscience, New York, N. Y., 1975.

6. K. Matsuura, S. Hattori, I. Noda, and M. Nagasawa, Optics and Laser Technology, 10, 237 (1978).

7. F. Suzuki, K. Hori, N. Kozuka, H. Komoda, K. Katsuro, Y. Takahashi, I. Noda, and M. Nagasawa, Polym. J., 18, 911 (1986).

8. Y. Takahashi, Y. Isono, I. Noda, and M. Nagasawa, Macromolecules, 18, 1002 (1985).

9. P. G. de Gennes, "Scaling Concepts in Polymer Physics," Cornell University Press, Ithaca, N. Y., 1980.

10. S. Yukioka, Y. Higo, I. Noda, and M. Nagasawa, Polym. J., 18, 941 (1986).

11. I. Noda, N. Kato, T. Kitano, and M. Nagasawa, Macromolecules, 14, 688 (1981).

12. I. Noda, Y. Higo, N. Ueno, and T. Fujimoto, Macromolecules, 17, 1055 (1984). 